\title{
Technical developments for accurate determination of amount of samples used for TOF measurements
}

\author{
Kazushi Terada ${ }^{1, \text { a }}$, Taro Nakao ${ }^{1}$, Shoji Nakamura ${ }^{1}$, Atsushi Kimura ${ }^{1}$, Osamu Iwamoto ${ }^{1}$, Hideo Harada ${ }^{1}$, Koichi Takamiya $^{2}$, \\ and Jun-ichi Hori ${ }^{2}$ \\ ${ }^{1}$ Nuclear Science and Engineering Center (NSEC), Japan Atomic Energy Agency (JAEA), Tokai-mura, Naka-gun, Ibaraki 319-1195, \\ Japan \\ 2 Kyoto University Research Reactor Institute, Kumatori-cho, Sennan-gun, Osaka 590-0494, Japan
}

\begin{abstract}
Activity determination of ${ }^{241,243}$ Am samples has been performed with two separate methods of calorimetry and gamma-ray spectroscopy. Decay heat measurements of the samples were carried out by using a calorimeter, and activities of the samples were accurately determined with uncertainties less than $0.45 \%$. The primary source of uncertainty in the calorimetric method is the accuracy of available half-life data. Gamma-ray detection efficiencies of a HPGe detector were determined with uncertainties of $1.5 \%$ by combining measured efficiencies and Monte Carlo simulation. Activities of the samples were determined with uncertainties less than $2.0 \%$ by gamma-ray spectroscopy and were concordant with those of the calorimetry.
\end{abstract}

\section{Introduction}

Accurate neutron capture cross sections of Minor Actinides (MAs) produced in fission reactors are required in order to promote the $R \& D$ of the nuclear waste transumutation system for MAs. However, there are gaps between required accuracies and current accuracies on relevant neutron capture cross sections [1]. Current accuracies are $6 \%$ for the ${ }^{237} \mathrm{~Np}$ capture cross section in the neutron energy region from 0.5 to $500 \mathrm{keV}, 8 \%$ for ${ }^{241} \mathrm{Am}$, and $10 \%$ for ${ }^{243} \mathrm{Am}$ from 0.5 to $1350 \mathrm{keV}$. On the other hand, required accuracies are $3 \%$ for ${ }^{237} \mathrm{~Np}, 2 \%$ for ${ }^{241} \mathrm{Am}$, and $2 \%$ for ${ }^{243} \mathrm{Am}$. Additionally, discrepancies exist between measured capture cross section data deviating from their uncertainty ranges. To meet the requirement, "Research and development for Accuracy Improvement of neutron nuclear data on Minor ACtinides (AIMAC)" has been started to improve the reliability of the neutron cross section data of MAs by a combination of neutron capture and neutron total cross section measurements. The purpose of the project is to reduce the uncertainties of the capture cross sections of ${ }^{237} \mathrm{~Np}$ and ${ }^{241,243} \mathrm{Am}$ to half of the present values.

It has been pointed out that uncertainty due to the sample mass is one of the factors contributing to the discrepancies of measured cross section data [2-4]. For neutron capture and neutron total cross section measurements, the uncertainty due to the sample mass affects normalizations and accuracies for measured cross section data. However, the sample mass is not identified in some cases, resulting in unrecognized systematic uncertainties. Therefore, as a part of the AIMAC project, we have developed the techniques accurately determining the sample mass by two different methods: calorimetric method, and gamma-ray spectroscopic method. As for

$\overline{\text { a e-mail: terada.kazushi@jaea.go.jp }}$ the calorimetric method, decay heat of the samples was measured accurately by using a calorimeter. Since Q-values associated with radioactive decays of MAs are well known, activities of the samples can be obtained accurately. In the spectroscopic method, gamma-ray emission probabilities of ${ }^{241,243} \mathrm{Am},{ }^{239} \mathrm{~Np}$ have been determined with high precision for accurately quantifying the amounts of the samples, by direct detection of emitted gamma-rays from the samples, with a planar type High-Purity Germanium (HPGe) detector [5]. An efficiency curve of the HPGe detector was obtained by combining measured efficiencies and Monte Carlo simulation. Activity measurements of the samples were performed.

\section{Calorimetric method}

Americium-241 undergoes alpha-decay with a half-life of $432 \mathrm{y}$, and ${ }^{237} \mathrm{~Np}$ (half-life of $2.144 \times 10^{6} \mathrm{y}$ ) is produced. Americium-243 undergoes alpha-decay with a half-life of $7370 \mathrm{y}$ and produces ${ }^{239} \mathrm{~Np}$, which is in radioactive equilibrium with ${ }^{243} \mathrm{Am}$ due to a short half-life of $2.35 \mathrm{~d}$ [6]. Two ${ }^{241} \mathrm{Am}$ samples with nominal activities of 480 and $950 \mathrm{MBq}$ and three ${ }^{243} \mathrm{Am}$ samples with nominal activities of 60, 120 and $240 \mathrm{MBq}$ were supplied from Khlopin Radium Institute for neutron capture and neutron total cross section measurements at the Japan Proton Accelerator Research Complex (J-PARC). These Am samples were prepared by compression molding of mixtures of $\mathrm{AmO}_{2}$ powder and $\mathrm{Y}_{2} \mathrm{O}_{3}$ powder into the form of pellets with a diameter of $10 \mathrm{~mm}$ and a thickness of $0.5 \mathrm{~mm}$, then the pellets were sealed in $\mathrm{Al}$ containers with a thickness of $0.1 \mathrm{~mm}$. Isotopic purities of these ${ }^{241,243} \mathrm{Am}$ samples were analyzed by thermal ionization mass spectrometry and alpha-ray spectroscopy. The isotopic purities of ${ }^{241} \mathrm{Am}$ in the ${ }^{241} \mathrm{Am}$ samples were more than $99.9 \%$, and isotopic contaminations of ${ }^{239} \mathrm{Pu}$ 


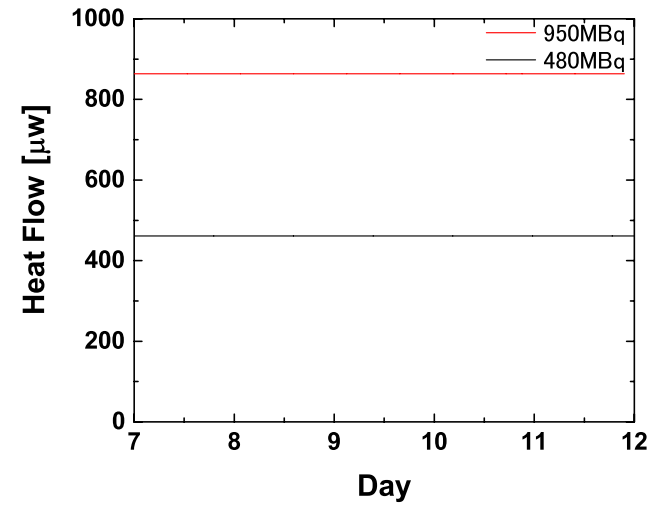

Figure 1. Decay heat of the ${ }^{241} \mathrm{Am}$ samples with nominal activities of 480 and $950 \mathrm{MBq}$.

were $0.09 \%$. On the other hand, isotopic compositions of ${ }^{243} \mathrm{Am},{ }^{241} \mathrm{Am},{ }^{244} \mathrm{Cm}$ and ${ }^{242} \mathrm{Cm}$ in the ${ }^{243} \mathrm{Am}$ samples were $97.36 \%, 2.39 \%, 0.26 \%$ and $1.00 \times 10^{-4} \%$, respectively. The impact of these impurities is described below.

Decay heat measurements of the ${ }^{241,243}$ Am samples have been performed with the TAM-IV calorimeter manufactured by TA Instruments. The precision of the calorimeter was $\pm 100 \mathrm{nW}$, and baseline stability was $\pm 200 \mathrm{nW} / 24 \mathrm{~h}$ in the certification sheet. For absolute calibration of the calorimeter, joule heat from a resistor of $10 \mathrm{k} \Omega$ with an uncertainty of $0.01 \%$ was measured while changing a current with a multimeter (DMM7510) having an uncertainty of $1 \mathrm{ppm}$. As the result, we have confirmed the uncertainty of the heat measurements of $200 \mathrm{nW}$. Gamma-ray energies emitted from the beta minus decay of ${ }^{239} \mathrm{~Np}$ are relatively high at around $300 \mathrm{keV}$ by comparing with those of ${ }^{241} \mathrm{Am}(60 \mathrm{keV})$. The ${ }^{241,243} \mathrm{Am}$ samples were held in the measuring container while sandwiched between two tungsten shielding disks with diameters of $20 \mathrm{~mm}$ and thicknesses of $12 \mathrm{~mm}$ in order to reduce the leakage of decay gamma-rays from the ${ }^{241,243} \mathrm{Am}$ samples to the outside of the calorimeter. The heat leakage due to the gamma-rays from the ${ }^{243} \mathrm{Am}$ samples were less than $0.1 \%$ according to calculations by using GEANT4 [7]. On the other hand, the gamma-ray energies emitted from the ${ }^{241} \mathrm{Am}$ samples are low $(60 \mathrm{keV})$, and heat leakage from the ${ }^{241} \mathrm{Am}$ sample was negligibly. To reduce the influence caused by air compression when the sample is inserted into the calorimeter, measurements of the ${ }^{241,243}$ Am samples were performed until the measured heat reached equilibrium. The measuring time for each sample was 12 days at least. Figures 1 and 2 show the heating values from the ${ }^{241,243} \mathrm{Am}$ samples. Uncertainties of the measured heating values for the ${ }^{241,243}$ Am samples were less than $0.26 \%$ taking into account fluctuations in measurement values and the baseline stability.

Activity $\left(A_{\text {cal }}\right)$ of the sample is defined as follows:

$$
A_{\mathrm{cal}}=\frac{H}{Q} k
$$

where $H$ the is measured heating value, $Q$ is $\mathrm{Q}$-value of radioactive decay for target nuclide, $k$ is correction factor for the impurities in the ${ }^{241,243} \mathrm{Am}$ samples. Qvalues for alpha-decay of ${ }^{241} \mathrm{Am}$ and ${ }^{243} \mathrm{Am}$ are $5637.81 \pm$ $0.12 \mathrm{keV}$ and $5438.1 \pm 0.9 \mathrm{keV}$, respectively [6]. For the ${ }^{241} \mathrm{Am}$ samples, contributions due to the impurities of

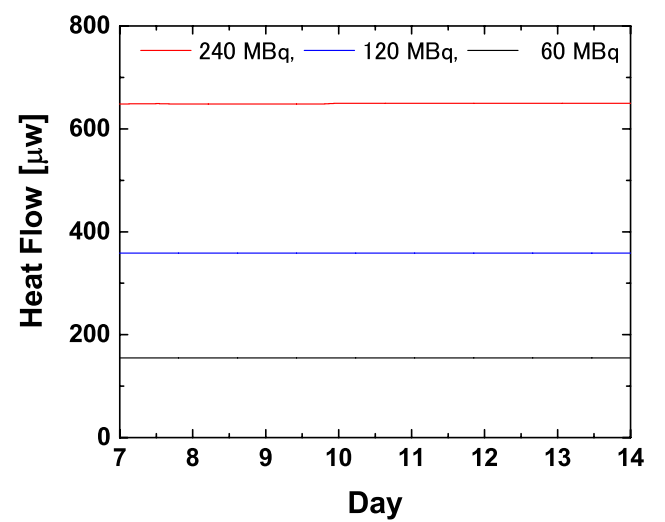

Figure 2. Decay heat of the ${ }^{243} \mathrm{Am}$ samples with nominal activities of 60, 120 and $240 \mathrm{MBq}$, respectively.

${ }^{239} \mathrm{Pu}$ were $0.08 \%$. The heat leakage caused by the gammarays of the ${ }^{241} \mathrm{Am}$ samples was negligibly. The decay heat from the daughter nuclide of ${ }^{237} \mathrm{~Np}$ does not affect due to a long half-life $\left(2.144 \times 10^{6} \mathrm{y}\right)$. On the other hand, impurities of ${ }^{241} \mathrm{Am},{ }^{244} \mathrm{Cm}$ and ${ }^{242} \mathrm{Cm}$ in the ${ }^{243} \mathrm{Am}$ samples have a great influence on obtained activities because their half-lives are significantly shorter than that of ${ }^{243} \mathrm{Am}$. Correction factor caused by these impurities were 0.4118 as for the ${ }^{243} \mathrm{Am}$ samples. Finally, activities of the ${ }^{241,243}$ Am samples were determined with uncertainties less than $0.45 \%$. The following points were taken into consideration for the uncertainties of the activities of the ${ }^{241,243}$ Am samples: (1) half-lives of the target nuclides ( ${ }^{241} \mathrm{Am}: 0.15 \%$, $\left.{ }^{243} \mathrm{Am}: 0.30 \%\right)$; (2) the uncertainties caused by decay heat measurements $\left({ }^{241} \mathrm{Am}:<0.04 \%\right.$, $\left.{ }^{243} \mathrm{Am}:<0.26 \%\right)$; (3) the uncertainties due to the Q-values $(<0.02 \%)$. The uncertainties of the half-lives of ${ }^{241,243} \mathrm{Am}$ were the most dominant uncertainty component.

\section{Gamma-ray spectroscopic method}

Gamma-rays emitted from the ${ }^{241,243}$ Am samples were measured with a planar type low energy photon HPGe detector (GLP-36360/13P4) having dimensions of $36 \mathrm{~mm} \times 13 \mathrm{~mm}$ manufactured by ORTEC. The detector was p-type Ge crystal with a Be window with a thickness of $0.254 \mathrm{~mm}$, where the cap-to-crystal distance was $7 \mathrm{~mm}$. The Ge detector was cooled with the X-COOLER III made by ORTEC. The energy resolution at $122 \mathrm{keV}\left({ }^{57} \mathrm{Co}\right.$ gamma-ray) was $0.6 \mathrm{keV}$.

Measurements of the ${ }^{241,243} \mathrm{Am}$ samples were performed with a source-detector distance of $150 \mathrm{~cm}$. Fullenergy peak efficiencies of the detector were determined by a combination of measurements for standard gammaray sources and Monte Carlo simulations using the PHITS code [8]. Two individual standard gamma-ray sources $\left({ }^{60} \mathrm{Co}\right.$ and $\left.{ }^{137} \mathrm{Cs}\right)$ supplied by JRIA having activities of $1.0 \mathrm{MBq}$ with uncertainties of $1.5 \%(1 \sigma)$ were used. Measurements of the standard sources were carried out for a long period of time to reduce statistical uncertainties in peak areas. The dead time was less than $0.2 \%$. Peak areas were obtained by simultaneously fitting a Gaussian, a skewed Gaussian and a smoothed step function. The skewed Gaussian shows contribution of incomplete charge collection. The smoothed step function indicates contributions from incomplete charge 
collection effects and Compton scattering of gammarays in the detector. X-rays from ${ }^{137} \mathrm{Cs}$ were used to confirm the reliability of the efficiencies at low energies. The efficiencies were determined with the obtained peak areas, the absolute activities of the sources and gammaray emission probabilities of the ${ }^{60} \mathrm{Co}$ and ${ }^{137} \mathrm{Cs}$ nuclides. For the standard sources, evaluated data of half-lives and gamma-ray emission probabilities were taken from the International Atomic Energy Agency (IAEA) [9]. Contribution from cascade summing was sufficiently small for the measurements made at $150 \mathrm{~cm}$. The efficiency curve was interpolated by PHITS calculations taking into account physical parameters of Ge detector. The details of the PHITS calculations were described in [5], so it is briefly described in the present paper. We used Monte Carlo calculations to determine the parameters of the HPGe detector (radius, length and dead layer thickness ) to match the measured efficiencies, and precise interpolation of the efficiencies was achieved. The PHITS calculations were in good agreement with the measured efficiencies with an uncertainty of $1.5 \%$ ranging from 60 to $300 \mathrm{keV}$.

Measurements of the ${ }^{241,243} \mathrm{Am}$ samples were performed as well as the standard sources. Dead time of the measurements were less than $6.9 \%$. Areas of $59.54 \mathrm{keV}$ ${ }^{241} \mathrm{Am}$ peak and $277.60 \mathrm{keV}{ }^{239} \mathrm{~Np}$ peak in the measured gamma-ray spectra were determined by the Gaussian fitting. Statistical uncertainties of the peak areas were less than $0.1 \%$. Activity of the samples is defined as follows by gamma-ray measurements;

$$
A_{\gamma}=\frac{Y}{\epsilon I_{\gamma} T}
$$

where $Y$ is the peak area of gamma-rays, $\epsilon$ is gamma-ray detection efficiency, $I_{\gamma}$ is gamma-ray emission probability, $T$ is the measuring time. We have preciously measured the gamma-ray emission probabilities of ${ }^{241,243} \mathrm{Am}$ and ${ }^{239} \mathrm{~Np}$ by combination of gamma- and alpha-ray spectroscopic methods with uncertainties less than $1.2 \%$ [5], and the measured gamma-ray emission probabilities of $59.54 \mathrm{keV}$ on ${ }^{241} \mathrm{Am} 35.64 \pm 0.46$ and $277.60 \mathrm{keV}$ on ${ }^{239} \mathrm{~Np} 14.34 \pm$ 0.16 were used for activity determination. Corrections of gamma-ray attenuation caused by the ${ }^{241,243}$ Am samples and the air between the samples and the detectors were made. The correction factors were ranging from 1.179 to 1.195 for the ${ }^{241} \mathrm{Am}$ samples and 1.037 to 1.048 for the ${ }^{243}$ Am samples. Finally, activities of the ${ }^{241,243} \mathrm{Am}$ samples were derived with uncertainties less than $2.0 \%$ by taking into consideration the following components: (1) the statistical uncertainties of peak areas for the $241,243 \mathrm{Am}$ measurements $(<0.1 \%)$; (2) the uncertainties of gamma-ray detection efficiencies $(<1.5 \%)$; (3) the uncertainties of gamma-ray emission probabilities of ${ }^{241} \mathrm{Am}$ and ${ }^{239} \mathrm{~Np}$.

\section{Results}

The activities of the ${ }^{241,243}$ Am samples were determined by two individual methods of calorimetry and gamma-ray spectroscopy. Figures 3 and 4 indicate the obtained results. It is noted that excellent activity determination became available by using the calorimeter with the uncertainties less than $0.45 \%$. Their results were in good agreements.

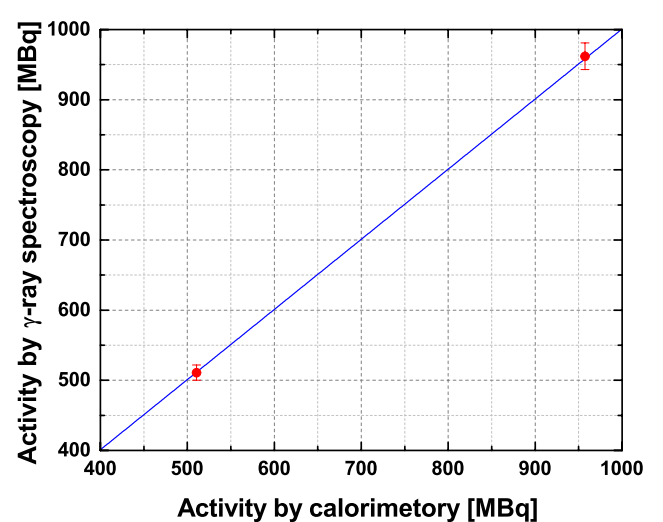

Figure 3. Activities of the ${ }^{241} \mathrm{Am}$ samples determined by calorimetry and gamma-ray spectroscopy.

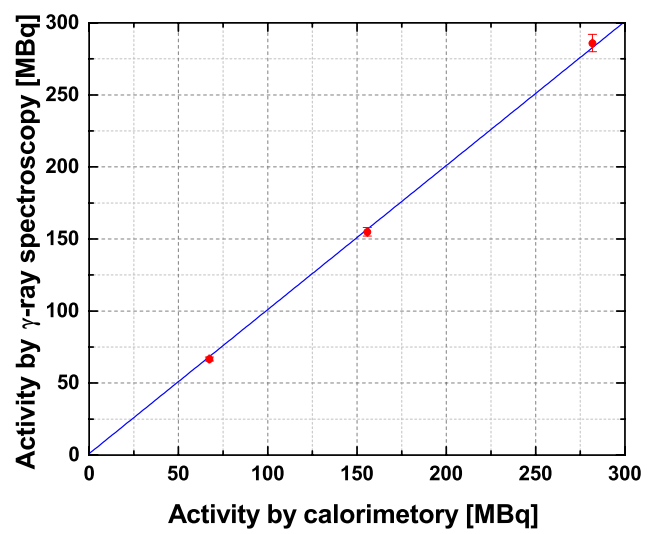

Figure 4. Activities of the ${ }^{243} \mathrm{Am}$ samples determined by calorimetry and gamma-ray spectroscopy.

\section{Conclusions}

We have developed the techniques for accurately determining the sample mass by: a calorimetric method, and a gamma-ray spectroscopic method. Decay heat of the ${ }^{241,243}$ Am samples was measured accurately by using the calorimeter with a precision of $200 \mathrm{nW}$, and thus activities of the samples were obtained accurately with uncertainties less than $0.45 \%$. In gamma-ray spectroscopic method, the efficiency curve of the HPGe detector was derived by combining measured efficiencies and Monte Carlo simulation. Activities of the ${ }^{241,243} \mathrm{Am}$ samples were obtained with uncertainties less than $2 \%$. The activities of the ${ }^{241,243} \mathrm{Am}$ samples obtained by the two separate methods were in good agreement, and highly accurate quantification of the ${ }^{241} \mathrm{Am}$ samples with an uncertainty approaching $0.15 \%$, limited by available half-life data, was enabled by calorimetry.

The author would like to thank the accelerator and technical staff at J-PARC for operation of the accelerator and the neutron production target and for the other experimental supports. Present study includes the result of "Research and Development for accuracy improvement of neutron nuclear data on minor actinides" entrusted to the Japan Atomic Energy Agency by the Ministry of Education, Culture, Sports, Science and Technology of Japan (MEXT).

\section{References}

[1] M. Salvatore et al., OECD NEA Report 6410 (2008)

[2] K. Hirose et al., J. Nucl. Sci. Technol. 50, 188-200 (2013) 
[3] H. Harada et al., Nuclear Data Sheets 119, 61-64 (2014)

[4] E. Mendoza et al., Phys. Rev. C. 90, 034608 (2014)

[5] K. Terada et al., J. Nucl. Sci. Technol. Published online: 26 Apr 2016

[6] Evaluated Nuclear Structure Data File (ENSDF). Database version of May. 2015. Available from: www.nndc.bnl.gov/ensdf/index.jsp
[7] S. Agostinelli et al., NIMA 506, 205-303 (2003)

[8] T. Sato et al., J. Nucl. Sci. Technol. 50, 913-923 (2013)

[9] International Atomic Energy Agency, Update of $X$ Ray and Gamma Ray Decay Data Standards for Detector Calibration and Other Applications, (2007) 